\title{
The Interstitial in Structural System in Architecture
}

\author{
Jinan Hassan M. AlAnbakya, Hadeel Saad razoki al-mur'ib \\ ${ }^{a}$ Uruk University, Baghdad, Iraq \\ ${ }^{b}$ Architectural Engineering Department / Al-Muthanna university \\ * jana_arch@yahoo.com
}

\begin{abstract}
Previous knowledge has shown a concept revealing different boundaries and relations of building spaces with its outer shell; which represent the boundaries of these spaces. This concept is called interstitial space which is based on creating an interlocution and communication within this space giving a new understanding of meaning and a new approach in architectural formation. This concept of knowledge description has varied as it is linked once to its nature or again to the built environment levels whether it's architectural or urban diverged within the academic and social context, as well as the blurring relationship nature of this space with the structural system of the building. Therefore, the problem state of this research, which goal focuses on, is highlighted as "the urge to investigate the concept of interstitial structure characteristics". Yet the methodology of the research is based on the descriptive and analytical approach consisting of three phases; phases one is to build a theoretical framework about the characteristics of the interstitial space in architecture; second is to conduct a practical study and identify several samples of buildings vary in their structural systems (traditional, structural and space); then analyze data and identify the conclusions of which the interstitial space grows and expands physically and expressively with the presence of the recent constructive systems and the lack of presence of the Interstitial spaced has led to spatial differentiation and closed toward inside especially those designed parametrically and reflect it in the other traditional and structural constructive systems.
\end{abstract}

Keywords: Interstitial space, structural System, building shell, form/structure.

\section{Introduction}

The word Interstice is belonging to Latinian word as "interstitium" as averb "interiacere"[1] . It has been formed from "prefix inter-“ called "between" it's mean the space between things, also comes framesets-"standing" which express about something stopped in the middle of the thing or a gape [2].There are many dictionaries identify the interstice such as in Arabic dictionary "The clossary of omnibus meaning, which called the interstice as "al-Khalil" means "obtuse" [3], While in the English dictionary and according to Cambridge dictionary the Interstitial is expressing the "space between the things and between events"[4] .Idiomatically, the word Interstice has come as "Interval" which look like old Chines word that indicate o the "Door" or "Entrance gate" [5].While regarded to the Architecture Steven Hool as a dialogue which works under tension, that based on many of conversations[6].From the other hand,the concept of the Interstice is similar with in-between concept.so this concept is signify of betweeness status in time and place[7] .The Interstice is considered as a case of in-between status, where one of the Interstice functional, is make anew relationship to achieve an interaction and 
relate between the contrasting parts[8] .So the Interstitial is space which mediates between two contradictionory situations, and the same time interaction as adialogue area which linking between spaces.

\section{Interstitial Space in Previous Studies:}

The scope of concept has been diversified in previous architectural Studies:

\subsection{The concept of Interstitial space}

is associated with natural, where the linking is come as a integrated spatial cavity in the formation of the Interstitial in the nature, where comes also to achive

a relationship of balance of the nature systems, through adding to the whole and subtracting from the whole such as the mountains are like the relationship of (adding to the whole) and the hills came as a subtraction from the earth (a relationship subtracted from all)[9]

2.2 The Interstitial has used in social theory to describe a different kind of operations that happened in cracks inside some of social structures. The benefit of the interstitial strategy is for change and equivalent transformation in society. The system of the organizational structure no matter how powerful it's with the help of the social in qualities, the Interstitial strategy doesn't come with the aim of sabotaging it. Thus the Interstitial is calls for social liberalization such as the bodies , women's shelter, social economy services and others. Therefore, it has a pivotal role in the patterns of social change and transformation[10]

2.3 The Interstice concept has shown in Academically as a benefit for a specialization to make it a closer through productive connection and possible interactions.

2.4 The Urban Interstice has considered the Interstice as urbun spaces and spaces outside official areas such as abandoned places and neglected places, therefor it's contradict the social and economic image.[11]

2.5 The Interstice concept has shown as waiting place, time gap, "in-between time-space" in urban environment such as a public wating place that be connect with other environment that people do their daily activity in it. So pointed to social Interstice roles in daily and urban life net.[12]

2.6 The Interstice is a part of landscape as a transitional space and apart of connective tissue. Harvard University has taken as a simple for interstice, so confirm that the interstice is a tight and small area which located between buildings and streets a winding paths, also the walls and doors of interstice area is considering within excesses surrounding the architectural continuation for the university. So the importance of the interstice is design and functionally[13] .

2.7 The researcher Dr. Ali AL-kafaji has made the interstitial in his thesis linked with hybridization strategy from the other hand this strategy open another space to negotiate to giving a meaning and questions of belonging and identity remain open for negotiation[14]

2.8 The Interstitials concept was related with the In-Between's concept through the linking and communicating. The interstitials concept is considering as important element in place making it the event that gives the place the vitality. So every place must be having three elements such as people, place and event. The element of people has the chance to bring the vitality to the space and the element of space is the physical environment which the people interact. As for the event is a connecting element in create a feeling among people towards this place, so it's consider important element[15] .

From the above, we conclude that there is a diversity in the interstitials concept on the level of the architecture and urban. This research is focusing on the nature of the interstitial space in Structural systems, So the problem of research and the goals is determining as:

* The research problem: the need to identify the nature of the interstitial space in the structural system.

* Objective of the research: determine the nature of the interstitial space in the structural system. 
* The research hypotheses: the nature and appearance of the interstitial space is differing from one structural system to another, and its diversity has shown in the vacuum structural system without traditional and structural.

* The research methodology: which depends on:

- building theoretical framework on the properties of the interstitial space, the formation nature and isolating the vocabulary and changes.

- practical study, applying the vocabulary of the theoretical framework to an elected sample of buildings with a construction system.

- analyzed finding and determine conclusions and the recommendations.

3. Theoretical framework: The Specialization of the Interstitial space in The Structural systems:

\subsection{The Prosperities of the Interstitial space}

3.1.1 The Interstitial space as an overlapping area between the Inner and Outer: The Interstitial is conceding as area of overlap between the things and the opposite of this things. The benefit of this interference is to provide an infrastructure for connections, where this infrastructure is providing a space- event connection with implicit communication[16], The Interstitial is characterized against spatial differentiation and absolute boundaries and replaces them with interlocking interaction to become a connective tissue where be a connecting with other formations a linkage tissue that create a place for imaginations, interactions and experiences[17]. As well as, it's conceding as aregion of spatial interferences and collisions between the contradictions. The Interference in The Interstitial is expressed through the (third part), which is must be defined on the one hand and controlled by the other. The interference is either be a partial through the third element or total, and The interference area has appeared informally as a specific barrier and a separator between the inside and outside, which is show as a separating wall or as interconnected link walls with the shape[18] . Therefor The interference is not just a separating site but rather an interference and intercalation. thus, it's be an indivisible insensitive unit[19]

3.1.2 Interstitial space as a Dialogic Structure: The pluralism is one of the interstitial methods. Interstitial is characterized by creating a dialogue between the two contradictions, so this creating a multiple moment individually, and the moment in time is consedring as an event and a thing to test it, while the moment in place is consedring as known place and a thing that we feel we live. The moment is the smallest unit in meaning as in pixel. The structure is formed by a series of pixilation where this image when we zoom it the image will be more clear and therefore we see this image clearty[20] . The Interstitial has the able to weld the variations, this means that any variations the interstitial will combine them. The Interstitial is conceding as experience (piece as concept), as apace with the possibility of connecting the things, and contextually meaning that derived from regional, cultural or historical content[21]. As an example of a structure the ornament in the mosque is considered as an integrated structure, due to that this ornament is a system that forms the mosque formal and mentel.

3.1.3 Interstitial space as a Design Structure: The Interstitial is one of a form of domination from the other hand, the interstitial is an experience that can creates its own dimentions based on the terrain it includes and it's gool is to reshape the whole. The benefit of the Interstitial space is to create a linkage between the user and what surrounds it. This space is provided a connection between one place to another, so is not just a normal space or avoid space. As well as this space has the ability to provide a form to the context through determine the place of the context by the relationship between the building and the outer space, where this context could be formed as long as there is an integrated spatial design with the context. The Interstitial space is either be openness with the context or be clouser. Also the another functional of the Interstitial space is giving a form to the context through a relationship between the void and the solid[22], where the void has dependent on 
the solid to knowing spatially. There are many shapes of the interstitial space such as a vacancy for all, as a space product after displacement or as intersection, absence of the shape by revoke it from the original context, or from the relationship between the solid/solid, where happened a space between two elements[23],also the other shape of the Interstitial space is apparent as a modules unites of the building where the benefit of this units' is to allow the building to resilience of spatial organization and is considered as a system that create a visual communication and physical with context[24].

\subsubsection{The Interstitial in bilateral formation (the Stricture/the Form):}

the Functional of the Interstitial: is to create an integrated and a balanced architecture (structurally and morphologically), where it's conceding as a field that the communications and interactions between the diodes happens on it.

First: Functional Formation: In this type the form is following the functional, while the structure is appeared as a technical, architectural element[25]. The form of the architectural origin has followed its functional rules, where focuses on durability of the architectural facility and the simplicity of the form that derived from the origin, so the ornament and formation which regarded impractical design does not take in the form of the architectural [26]. Therefore, the ideal architecture form is following what the building owner requests of special design for the building as what we called "the data" addition to look at the climatic and the environment conditions when designing[27]. Thus the functional formation in this type we conclusion that the interstitial field and the interaction between the (form /structure) is weak due to the less interaction of the structure with the form where the building is performing its structural function more than aesthetic function. Thus it's diving a simple reading and less richness in meaning.

Second: Symbolic Formation: When The Interstitial field has appeared in the relationship between the form and the structure, this field has led to weak in the boundaries between these dualism, so the communication and overlap between has become more available. The essence of the structure and it's effecting on the form also the effect of the form on the structure all these are giving to the structure an aesthetic aspect addition to its functional aspect which is carrying the building. The existence of the symbolic meaning in the structure and giving the architectural structure a symbolic semantics which means (a message encoded with meaningful where the creative architect has formulated it through constructive vocabulary, and appearing it to the recipient) are enriches the design idea to read the building completely. The structural representation is divided into two unequal groups of their use:

A- The Big Group: this group has contained the natural things that found in the word such as, the Eidem project is a huge industrial ecological complex in England, that specialized by having many of domes, every domes are simulates a biological environment that containing varieties of plants from different part of the word. The domes have a hexagonal structure and an enlarged scale of a honey bee hive, as in figure (1)[28]

B- The Small Group: in this group as contain the things that manufactured by humans and it's considered as an inspiration source. Such as, church of the Autostrada in Florence, this church was built to commemorate the Italian workers who died while working in the construction of the highway, according to the architectural and users they had been considered this church as a hat of the Bedouins from the ancient Israelites, who are from the general travelers who they led this church in the past fig (2) [29]

Third: Mathematically Engineering Formation by Using the Computer: in this type of formation is create a new form and new design that different from the traditional methods. All these is coming through design techniques and the development of the computer and its programs. The important mathematically way which is adopted by the interstitial in the building formation is the Algorithm. Algorithm and the Interstitial: The Algorithm is a series of successive logical steps and using it to solve a specific problem, the algorithm is considered as the idea behand any acceptable logical program, the name of the Algorithm is coming according to "Alkhawarizmi" scientist who had created it in the ninth century, and the Algorithm is the using word in Latin and European language[30]. The Algorithms and computers are creating a different output each times when inters different data, so the relationship between the Algorithm and outputs is similar in the field of DOI:10.52113/3/mjet/2021-9-1/06-19 
calculator and thus is similar in the architecture [31]. fig (3), therefore, it had approved of the Algorithm to define a problem and then follow a specific number of steps by using the computer and depends on the programs and math metical charts. From the other hand, the Algorithm were recently invested in the architecture as a design methodology and had adopted as a basic link between two system (order/disorder). Where instead of consideration the system and the randomness as a two distinct cases or as an opposite cases ideality, it's can be the benefit bigger through studying the process of changes each of (order/disorder) to another case, which be an intertwined folds, cross lines and broken blocks that reveal a complex patterns that aren't optically incomplete as if they are in a continuous motion. The Interstitial has shown in the place that happened the transformation between two systems, so The Interstitial (betweeness space) is producing a plurality in the directions of reading the form[32], There is many of buildings that designed according to the Algorithm system such as the British National Museum of Norman Foster and its partners, and the booth of the serpentine exhibition for architectural Toyo Ito , and also has shown in Beijing international stadium for the architects Herzog \& de Meuron, and the ocean north music and art center for architect Jyvaskyla [33] fig (4-5-6-7).The technical parametric designs is consedring as one of the methods of modern designs to produce the architectural form that depends on the Algorithm and the Mathematical processes through using the computer and it's working by inserting many of determinants of the building to be designed from length, width, height, weight, material and used symbols. It's a dynamic design which depends on the repetitive units in infinity formations by simulating the nature and understanding the structural system[34] . the fig (8) is declared the method of the Algorithm, after we are determining the problem and the objective then we are starting to work on the Algorithm steps with order under the principle of (If-Then), and can be written in many programming languages including (Java \& $\mathrm{C} / \mathrm{C}++$ ) and the results are accurate but the language are difficult to write and understand, so it's using the Pseudocode which represents a suitable medium for the Algorithm[35] .

3.2 The Structure System: Structure is the part that receives the influencing force on the building and transfers it to the ground. Or it's means that the architectural designer uses it to appear the form, and from the other hand, there is no living organic system without the Structure. Although that the Structure not being the building but it's make it possible. As the architecture is not the Structure, but it depends on the structural elements in achieving its objective[36] . The Structure of the building is consisting of a structural element where the designer is using it to realizes his idea. The benefit of the Structure is to determine the space, creating the units, suggest the movement or even develop the configuration and the model. Thus, the architectural form is connecting with the elements, which create the architectural form and determine its quality

\section{[37] The Type of Basic Structural Systems:}

3.2.1 Load Bearing Wall System: This type is one of the oldest types of the traditional construction systems in building with bricks, stone or concrete blocks. In this type also the loads and the weights are reported vertically to the basic of the building fig (9).

3.2.2 Skeleton System (Beams and columns): The system in this type consists of vertical elements (columns) and the horizontal elements (bridges), where the structural framework is providing a flexibility in the design of the built space[38] fig (10).

3.2.3 Space Frame System: This system is having a space frame and shell structures. Where this system had been apparent due to the technological development in materials, methods of implementation or construction system used. There are three interstitial structure systems which is:

A- Space frame: in this structural system has consist of a vacuum configuration where it's outer surface configurations has been geometrical. This system can be based on the pillars and the bearing walls. There is no needed to have a support element to help the vacuum element because they are having the ability to support themselves. The structure in this system is consist of repeated units which can be triangular, square, hexagonal or eight shape, and 
the role of this type is not just using in roofing but also include a single architectural and structural formation or columns with the ceiling and be in flat, oblique or curved shapes fig (11).

B- Shell Constructions: This thin-shell structures are consisting a short solid units and when this structures are designed it's needed to accurate arithmetic and detailed methods, which having the ability to withstand the force that keep the force in the direction of rotation and vertical direction to control them easily. The Shell Constructions are consisting of curved surfaces of thin thickness. This surfaces has the ability to resist the loads by the pressure, shear and tensile stresses. Its efficiency is being curvature and resistance to twisting where be double or single curvature and may to create a vacuum to achieve formative enrichment.

C- Flexible Construction Systems: That allow to growth and replacement according to changing needs and functions, such as tents.

\section{Description of the Selected samples:}

First sample (A): The first simple is represented of Beit AL-Raouf Mosque in Dhaka city, Bangladesh is considered as distinguished projects that the design won of the Aga khan award for the architecture 2016, the mosque area is $754 \mathrm{~m} 2$ and the project had been designed in 2006 and had completed in 2012. This project is specialized by simplicity and using a local building materials, ventilation and natural lighting in addition to this place is considering as a gathering place for people and children. Fig (12), as well as, this mosque is distinguished by using new additions in the design and in the building of the mosque. The sample is represented by its structural system with a load bearing wall and the building material which is the brick. From the other hand, the form of this simple has comes from the function of the same building and the structure has comes also according to the function. There is a simple opening in the design of this simple into outside and it's designed as a closed structure with the external context.

Second sample (B): The second simple is represented of Stansted Airport Terminal in Britain which designed by Foster office and its partners in 1991. This building is depending on structural system (the columns and bridges) fig (13). The form of this building had been chosen from forces and trees to inspire this building where this structure had been chosen due to the human are understand this structure as a high-buildings and represent them as the forces shapes. In this building, the structure is connected the outside and inside and the columns (trees) is consist of four iron pipes on square network that linked together with some bridges to be two-way frame and its form is be a pockets for ribs. The distance is about $36 \mathrm{~m}$ between the ribs so this giving a magnificent form then the forces form, thus a large number of columns are inspired from idea of the forces and plans.

Third sample (C): This simple is represented of the King Abdullah Center for Petroleum Studies and Research, this center is a global research center concerning the research of the oil, energy, environment and future policies. The design of this project is consedring of the barramic designs, and the center is executed by Saudi Aramco Company and it's located in the KSA in AL-Riyadh, and designed by Zaha Hadeed in 2016 fig (14). The project was designed as irregular hexagonal units. In this design, the horizontal and vertical line are not appearing easily because the inclined angle in its design. As well as, there is a small window to reduce the sunlight and energy consumption. The inner courtyards and the triangular openings in this building are bring the natural air and light to the interior. The design idea had been got from the hexagonal cells of the foliage as an affirmation of the interconnection philosophy, in addition to using the (yard) is to consider of the region's ritual, it's heritage and also to study of the shaden areas, gardens and the general site. The benefit from the (yard) is to host the meetings and enhance the exchange process because it's considered as an opening space. The building was chosen because the architectural structure is depending on the space frame system, where is consisting of hexagonal cell, which this cells are determined the outer of the surface building and its parts are based on the columns and the supporting walls.

\section{Method of measurement and data analysis, and measurement variables:}


The practical phase of the theoretical framework came through the direct recording of detailed single, and the method of statistical analysis will be adopted numerical to analyze recorded data that record the degree of compatibility with its detailed variables, where the degree of compatibility has been classified to:

- $\square \quad$ weak or non- degree of compatibility

- $\square$ Medium degree of compatibility

- $\square$ High degree of compatibility

6.Discussion and analysis of results: When applying the measurement scale to the three elected samples for practical study, the result that are related to each individual of vocabulary of theoretical framework first, then the degree of compatibility of the variables that related to the Interstitial space in in Structural System in Architecture, as in Table.1

First: The Result for First Single that Relates of the Interstitial Shows as an Overlap Zoon Between the Inside / Outside: The first sample (A) from the type of the Load Bearing Wall structural, the Interstitial field was more narrow and less interaction between the inside and outside, so we note that it's characterized of separation and isolation. While the sample (B) from the type of the architectural Skeleton System structure there is an expansion in the Interstitial field between the inside and outside to be overlap of the partial, so we note it's more openness towards the outside it's characterized by the extension of the roofing, the transparency of the glass and the use of the same shapes of columns and its symbolism in the interior and exterior. The sample (C) it's kind of space system which is of the type the structural space system with space frame, so expands, therefor the interference was total through merge the roofing with wall. The Interstitial field is more expands in the sample $(\mathrm{C})$ to indicate to the characteristic of the separation and communication.

Second: The Result for Second Single that Relates of the Interstitial shows as a Dialogue Structure: The first sample (A) which kind of the Load Bearing Wall structural of this type is focusing on the sensory intellectual dialogue, where the Interstitial has appeared as a spiritual structure that linking the earth with the sky and creating a dialogue energy between them. Therefore, the Interstitial had been shown as a dialogue structure through linking the bilateral more intellectually than formally, thus achieving a weak degree of compatibility. the sample (B) which is kind of the architectural Skeleton System structure, where it's had achieved an intermediate degree of compatibility. The Interstitial has appeared as a spiritual structure. it's had shown as adialogue to find convergence between the entities as it's an overlap area of the disparate parts by linking the meanings and also linking the regional contextual. The $\operatorname{sample}(\mathrm{C})$ which is kind of the structural of space system with the space frame, so we note in this sample that the power of the dialogue energy of the Interstitial between the parts is large then the other samples, therefore that's led a dialogue between the temporal segments that formed a coherent integrated structure that giving to the building the thought and the form through its modules which working on linking the regionally, heritage and culturally of context, thus achieved a high degree of the compatibility.

Third: The Result for the Third Single that Relates of the Interstitial shows as a Design Structure: The first sample (A) had shown the Interstitial as a closed design structure through the hard wall in it. While the second sample (B) and third sample (C), the Interstitial had shown as an opening structure, where the sample (B) is regarded a medium compatibility while the sample (C) is regarded a high compatibility in its appearance as an opening intermediate structure with its continuity and design transparency.

Forth: The Result for the Forth Single that Relates of the Interstitial Features: The results of the Interstitials features which is (communicative, hybrid and dynamic) all there are represents of many of samples, such as in sample (A), the communicative feature had shown through the sensory communicative more than the other features, so the value in this sample has become weaker. In the sample (B), which is the kind of the skeleton system structure has achieved an average degree of compatibility, where the feature of the communicative (Intellectual and Formal) and the dynamic has appeared through its movement, vitality and openness into the outside. The sample $(\mathrm{C})$, which is the kind of the structure of space frame system, we note of this sample the high degree of the compatibility due to all the Interstitial feature had appeared from the term of intellectual and formal communicative, hybrid and dynamic. 
Fifth: The Result for the Fifth Single that Relates of the Interstitial Formation Between the Structure / Form: The results of the Interstitial formation between the structure and form (Functional, Symbolic and Mathematically), the sample (A), which is the kind of the structure bearing wall it's recorded a high compatibility in the functional formation from the term of simplicity in form, durability and focus on the functional benefit. While the second sample (B) and third sample (C) has recorded a low compatibility. In the term of the symbolic formation, the second sample (B) has recorded a medium compatibility and the building was designed from the Symbolism of the forest and trees. The third sample (C) was recorded a high compatibility which has been taken the Symbolism of the foliage as well as the site and heritage context of the city. And from the Mathematical formation the sample (A) and sample (B) has recorded a weak compatibility, while The third sample (C) was recorded a high compatibility due to its parametric designs that have been Mathematically dependent on the logarithms.

\section{Conclusions:}

1. By analyzing the research samples the Interstitial is in creation anew relationship between the entities that collide with each other. So this relationship is providing aspectual, intellectual and material dialogue between the contradictions thus it's be an interaction between them.

2. The Interstitial space has appeared either from the shape of a visible zoon which characterized by the spatial force, or be in the form of an invisible zoon.

3.The Between Interstitial space is a dynamic, hybrid and stream lined space that is more noticeable in the parametric structural system.

4. The Interstitial field is become bigger and expanded when there is an Interstitial between the contradictions and openness to each other. So the Interstitial field is expending physically and expressively whenever the opening is become totally between the contradictions and appears when the building is designed on the space frame structural system.

5. The Interstitial is either be openable structure when it's be more fluid and stream lined and that's what apparent in the bearing wall and space frame structural system, or be a closed structure when the relationship between the contradictions is more rigid so this appears in the Load Bearing Wall System.

6. The Interstitial produced a multiple reading of the building, that accrue a change and transformation between two contradictions and made them as a host contradiction with overlapping form, where it showed in the space frame system more than skeleton structural and Load Bearing Wall System.

7. The lack of presence of the Interstitial spaced has led to spatial differentiation and closed toward inside. As in the Load Bearing Wall System.

8. The Building with the Load Bearing Wall System have a less overlapping due to the lack of the Interstitial spaces. Which provide the flexibility between the relationship while the overlapping in the parametric space frame system is more strength 9. The Dialogue feature which is one of the properties of Interstitial has provided the idea of Incubation of the structural system. Where the parts of the structure system have become more coherent in the space frame and skeleton structural system, while many parts of it differentiation between each other in the Load Bearing Wall System.

\section{REFERENCES:}

[1] http://www.dictionary.com/browse/ interstece

[2] http://www.merriam-webster.com/dictionary/interstece

[3] http://www.almaany.com/ar/dict/ar-ar

[4] http://dictionary.cambridge.org/dictionary/english/transition

[5]Batchlor,R,2012, "A taste for the Interstitial translating space from Beijing to London in the 1720s" "Sabean 3692_6208-055-P3-0141pass-r03.inddi, chapter foorteen,p.284

DOI:10.52113/3/mjet/2021-9-1/06-19 
[6]Mouch,D,2009,"Magnifying the Interstice:exploring the dialogue between architecture's in-betweens"Master thesis of architecture in the school of architecture and interior Design ,college of Design,Architecture,Art and Planning,University of Cincinnati, p.17 [7]Batchlor,R, 2012,"A taste for the Interstitial translating space from Beijing to London in the 1720s" "Sabean 3692_6208-055-P3-0141pass-r03.inddi, chapter foorteen,p.284

[8]AL-Anbaki,J,H.,2013,"In-Between Space in Architecture:A Study in The Nature of Active In-Between Space",Master thesis of department architecture, University of technology in Iraq, P.90.

[9] Mouch,D, 2009,"Magnifying the Interstice:exploring the dialogue between architecture's in-betweens"Master thesis of architecture in the school of architecture and interior Design ,college of Design,Architecture,Art and Planning,University of Cincinnati, p.18.

[10] Wright,E,2010,"Envisioning Real Utopias". London: Verso,p.228-230, http://www.aacademica.org/erik.olin.wright.

[11]Pamela.S,Joanne.H,2009,"TheQualitiesofInformalSpace:(Re)appropriation within the informal, interstitial spaces of the city". Proceedings of the Conference, University of Brighton, p1-4.

[12] Kärrholm,M. , Sandin,G. ,2011,"Waiting Places as Temporal Interstices and Agents of Change", Published articles, the Department of Architecture ,Lund University, p1-11.

[13]Spiegelman,K,A,2002,"LANDSCAPE:HARVARDPLANNING ANDREAL ESTATE", Copyright Harvard Planning and Real Estate. All Rights Reserved. , p5-7.

[14] Al- khafaji,A,M,2008, " Other in Architecture ", doctorate of philosophy in architecture,University of technology in Iraq, P.93.

[15]Combs,D,2003,"architecture and the interstitial :between space and event" Master thesis of architecture ,college of Architecture ans Planning Muncie, Indiana,University of Ball State, p.38.

[16]Combs,D,2003,"architecture and the interstitial :between space and event" Master thesis of architecture ,college of Architecture ans Planning Muncie, Indiana,University of Ball State, p.6.

[17]Mouch,D,2009,"Magnifying the Interstice :exploring the dialogue between architecture's in-betweens"Master thesis of architecture in the school of architecture and interiorDesign,collegeofDesign,Architecture,ArtandPlanning,University of Cincinnati,p.40-48

[18] Adnan,M,M,1997," The dialectic of the relationship between inside and outside in architecture",Master thesis of department architecture,University of technology in Iraq, P.26.

[19] Al-khafaji,A,M,2008, "Other in Architecture ",doctorate of philosophy in architecture,University of technology in Iraq, P.60.

[20]Nicolas,P,2007,"interstitianMultiplicity"www.le-commun .fr,p.2

[21] Combs,D,2003, "architecture and the interstitial :between space and event" Master thesis of architecture ,college of Architecture ans Planning Muncie, Indiana,University of Ball State, p.18

[22] Combs,D,2003, "architecture and the interstitial :between space and event" Master thesis of architecture ,college of Architecture ans Planning Muncie, Indiana,University of Ball State, p.6,18

[23]AL-Anbaki,J,H.,2013,"In-BetweenSpacein Architecture:A Study in The Nature of Active In-Between Space",Master of department architecture,University of technology in Iraq, P.70.

[24]AL-Anbaki,J,H.,2013,"In-BetweenSpacein Architecture:A Study in The Nature of Active In-Between Space",Master of department architecture,University of technology in Iraq, P.30.

[25] Wais,A,A,2008, " The effect of the material pillar of technology (materials, construction and origin) on the architectural form ",doctorate of philosophy in architecture,University of technology in Iraq, P.13-14.

[26] http://jehad-alkhandq.blogspot.com/2014/09/Functional-theory-in-architecture.html

[27] Wais,A,A,2008, " The effect of the material pillar of technology (materials, construction and origin) on the architectural form ",doctorate of philosophy in architecture,University of technology in Iraq, P.13-14.

[28] Charleson, A, 2005,” Structure As Architecture ”, Elsevier, Architectural press , New York , p(190-192).

[29] Charleson, A, 2005," Structure As Architecture ”, Elsevier, Architectural press , New York , p(198-199).

[30] Ayman ,Raes, Mohamed,2012 "Evaluation of Some Computer Programs Aiding the Architectural Design Process", Master Thesis, Department of Architectural Engineering, Faculty of Engineering, Shubra, Benha University,p-26

[31] Terzidis,K,2006,"AlgorithmicArchitecture”, wiley publishing, Inc,ISBN:978-0-470-37548-8, p.26-35

[32] Al-khafaji,A,M,2008, "Other in Architecture ",doctorate of philosophy in architecture,University of technology in Iraq, P.89.

[33] Terzidis,K,2006,"AlgorithmicArchitecture”,wiley publishing ,Inc,ISBN:978-0-470-37548-8, p.11

[34]Swidan,A,H.,2015,"Parametric concept and its applications in interior design and furniture",publication in International Design Magazine,Vol 5,No.3, , P.2-4.

[35] Ayman ,Raes, Mohamed,2012 "Evaluation of Some Computer Programs Aiding the Architectural Design Process", Master Thesis, Department of Architectural Engineering, Faculty of Engineering, Shubra, Benha University,p-26

[36] Wais,A,A,2008, " The effect of the material pillar of technology (materials, construction and origin) on the architectural form ",doctorate of philosophy in architecture,University of technology in Iraq, P. 3. 
[37] Mando,H,A.,2011," The Mutual relationships between the structural form and architectural forms in free curved form",Master of department architecture,AL-Baath University in Syria, P.5.

[38] Al-khafaji,A,M,2016, "Tectonic in Architecture ", publication in International Design Magazine,Vol 30,No.1-2, P.131-133.

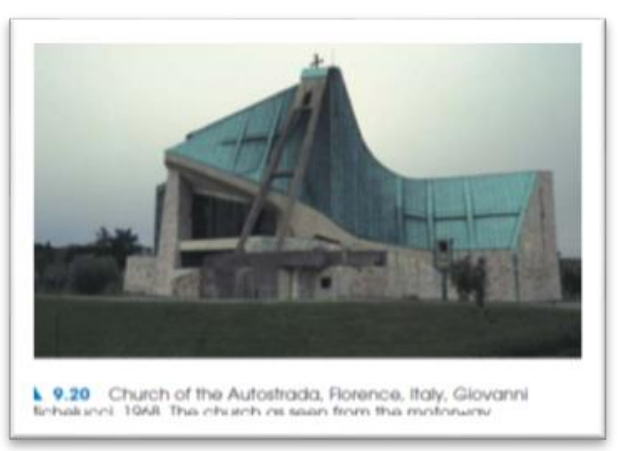

Figure.1. Eden project

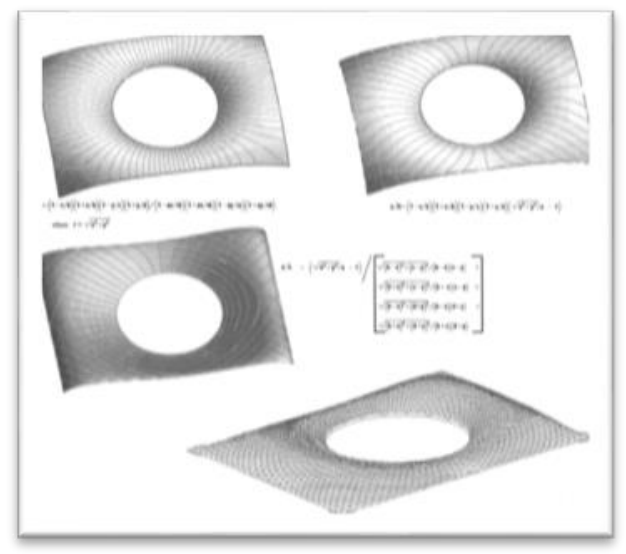

Figure.4 . the British National Museum of Norman Foster and it's partners

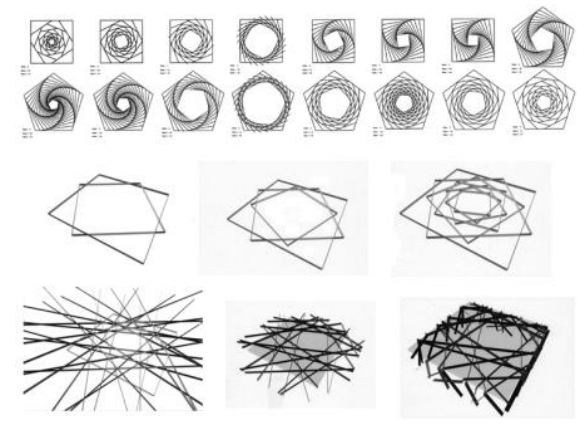

Figure.5. Booth of the serpentine exhibition for architectural Toyo Ito
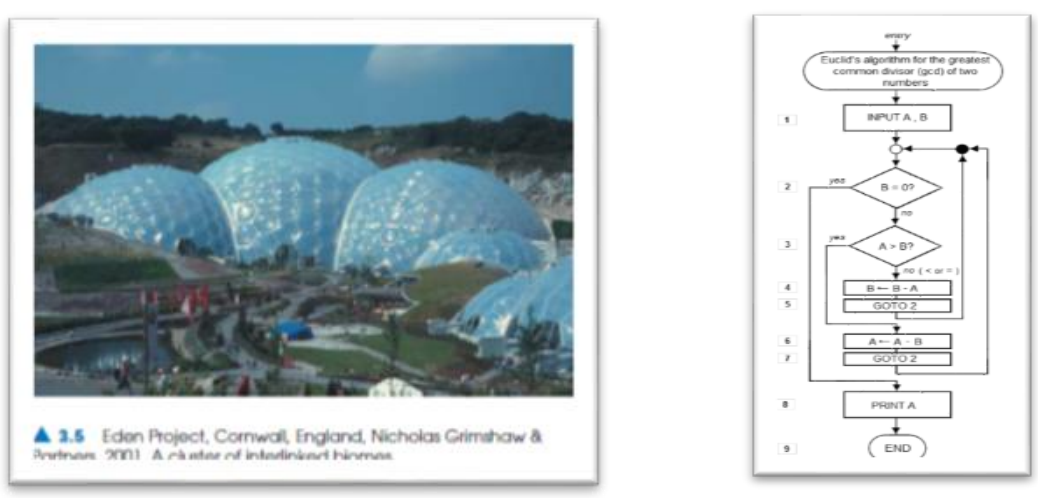

Figure.2 . Church of the Autostrada Figure.3.A simple idea of logarithms
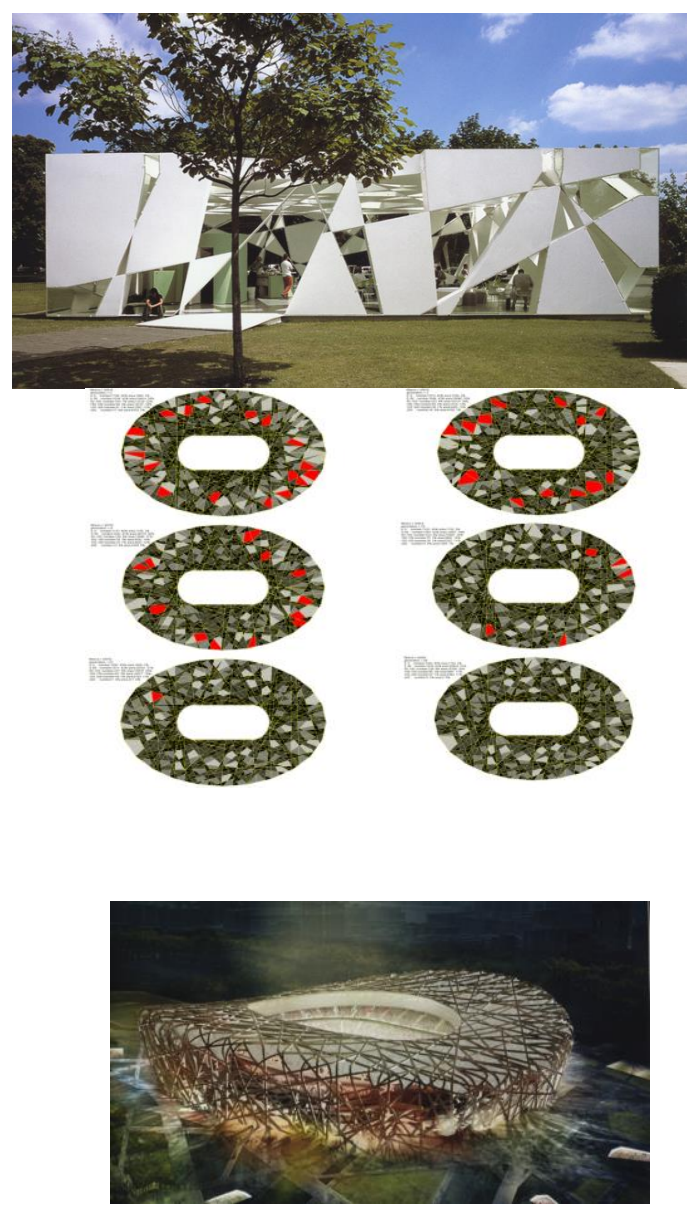

Figure.6 Beijing international stadium for the architects Herzog \& de Meuron Interstitial nodes 

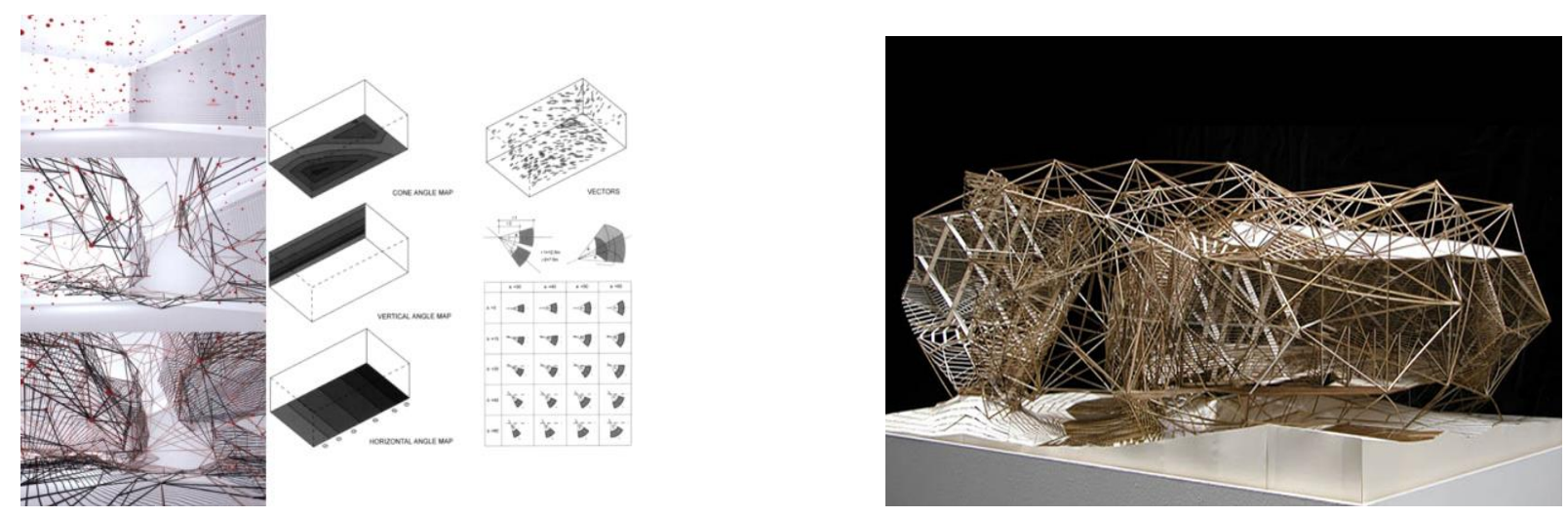

Figure.7 Ocean north music and art center for architect Jyväskylä
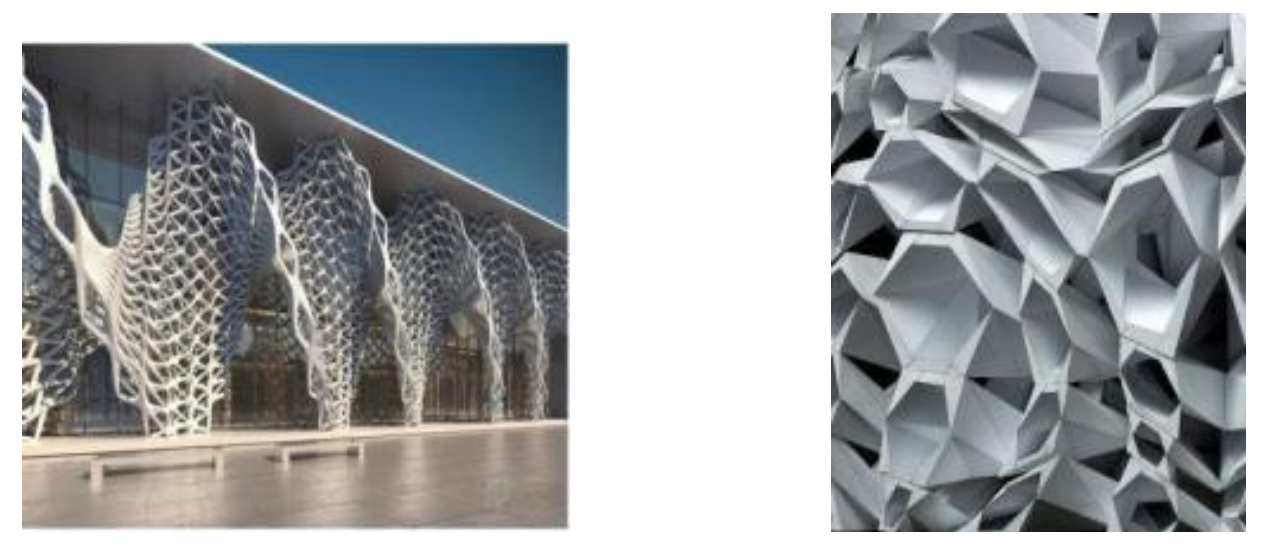

Figure.8 Parametric units shown Simulating nature and understanding the structural systems that underlie the structures of forms

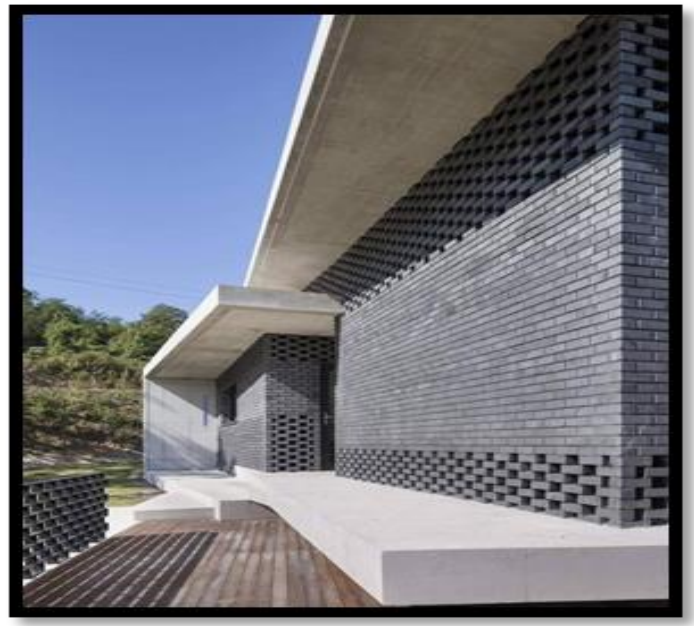

Figure.9 . Load Bearing Wall System

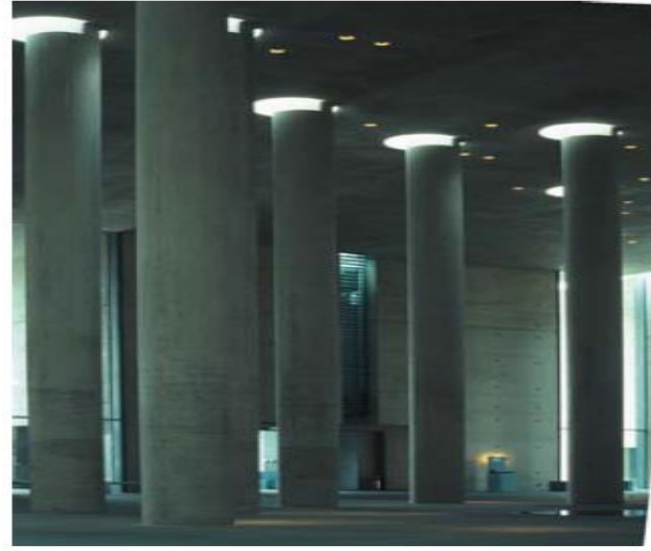

A.13 Condolence hall columns.

Figure.10 . Skelton system (columns\&brige) With abricks 


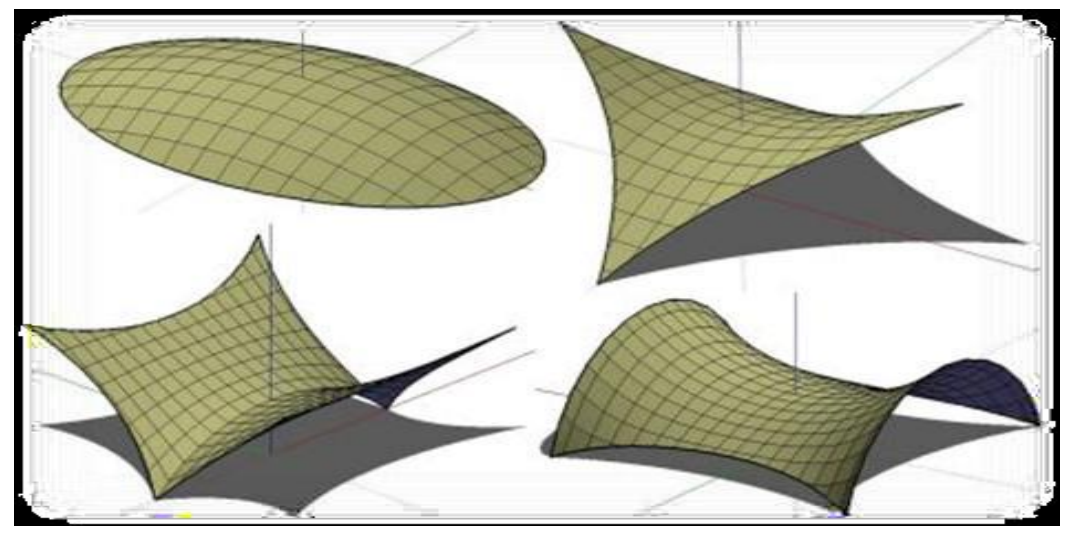

Figure.11 Space frame system
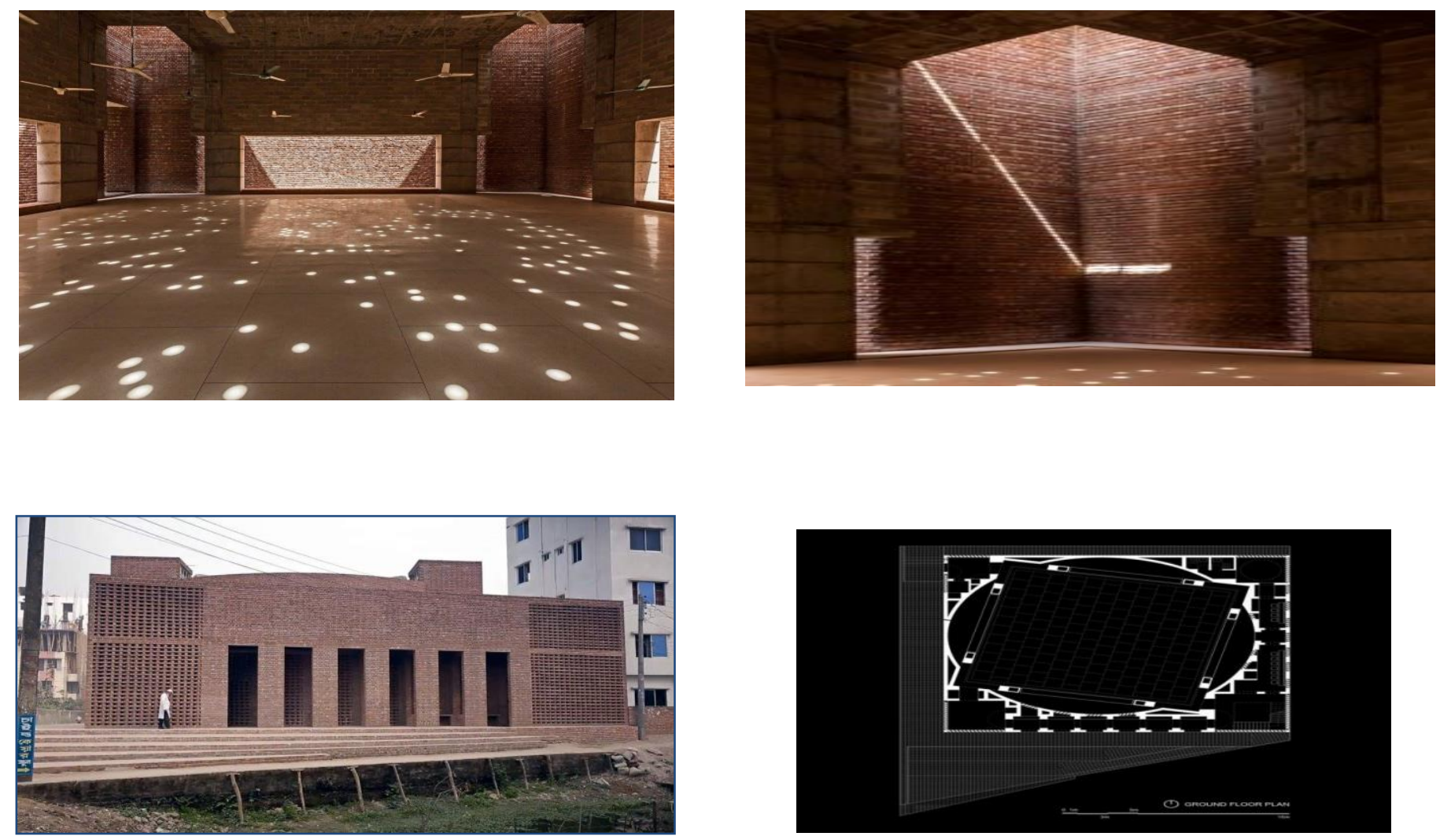

Figure.12. Beit AL-Raouf Mosque in Dhaka city, Bangladesh the design won of the Aga khan award for the architecture 2016

http://www.albayan.ae/five-senses/culture/2016-10-03-1.2726835 

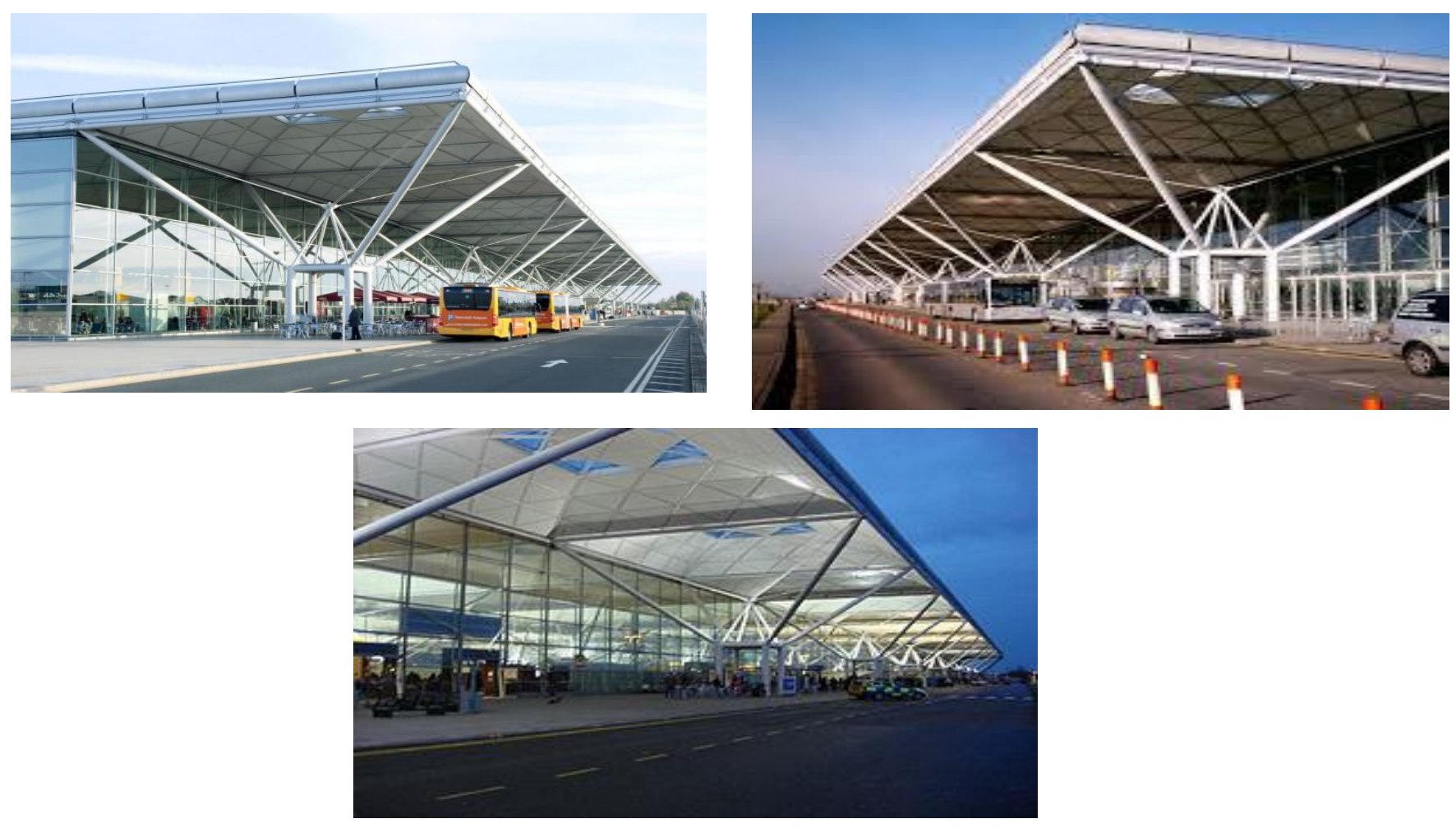

Figure.13. Stansted Airport Terminal in Britian which designed by Foster office and it's partners in 1991
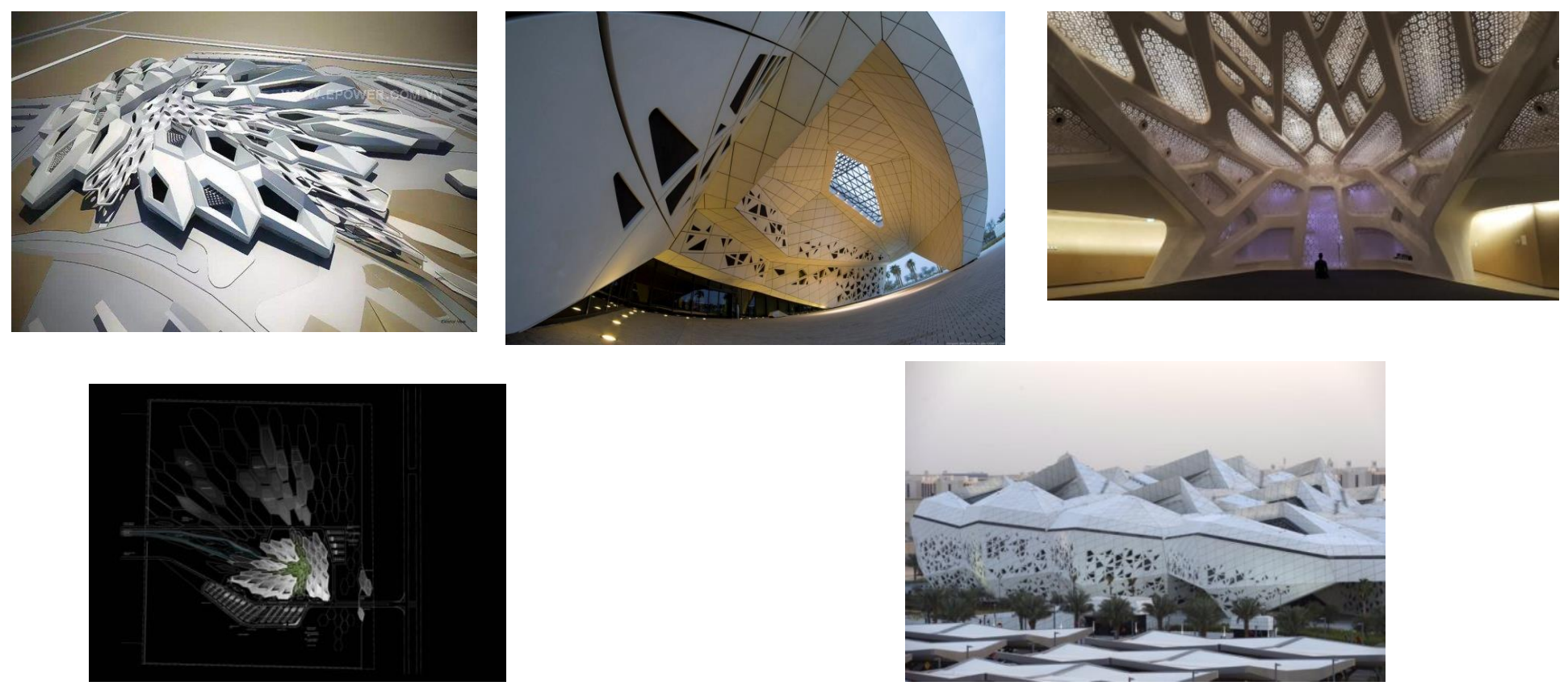

Figure.14. the King Abdullah Center for Petroleum Studies and Research by Zuha Hadeed in 2016 https://www.almrsal.com/post/327211 
Table.1 Comparing results in terms of the secondary variables between the three samples elected and the degree of compatibility of each variable.... Preparation: Researchers

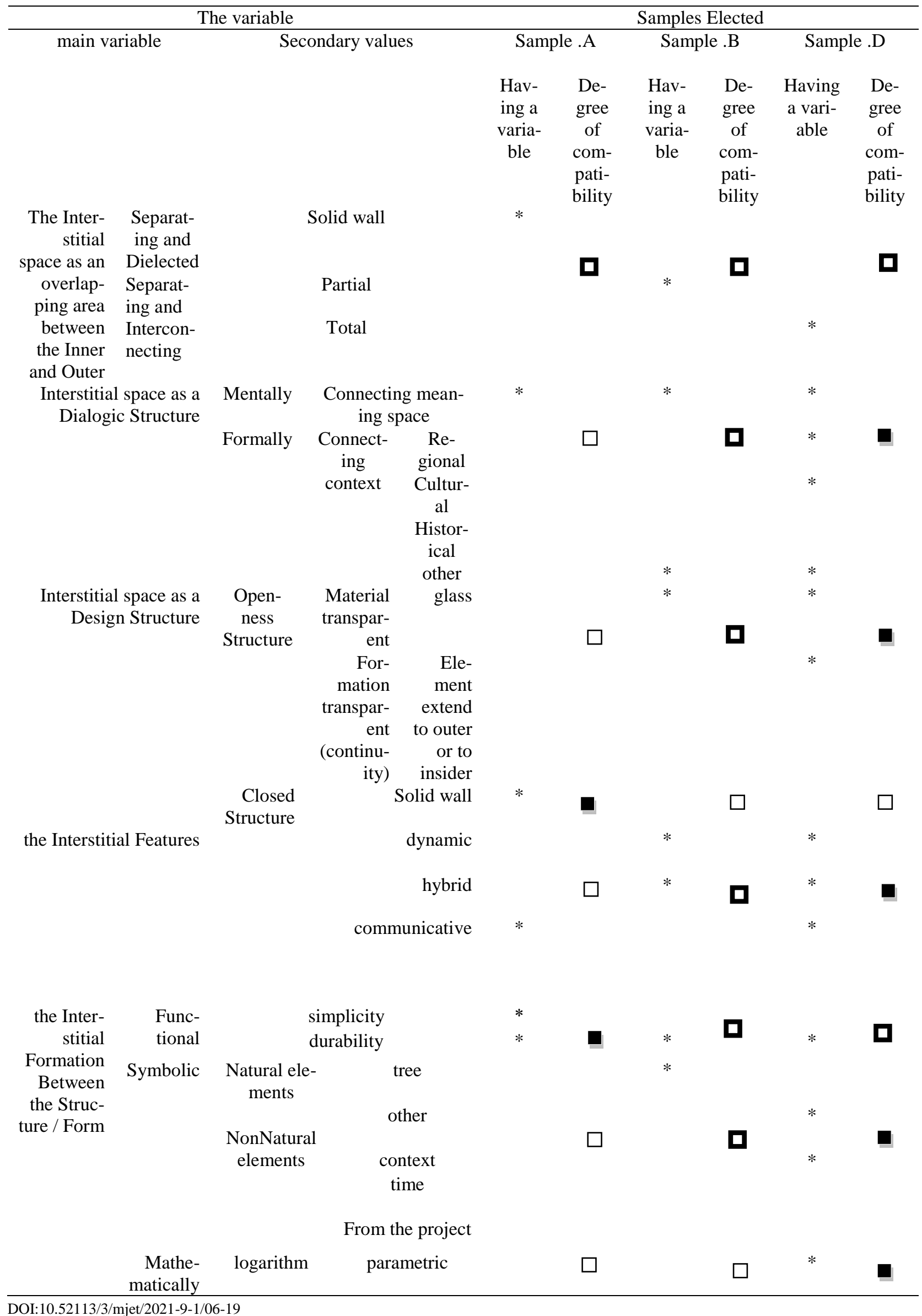

